\title{
Application of Modified Artificial Fish Swarm Algorithm for Optimizing Association Rule Mining
}

T. Bharathi* and P. Krishnakumari

RVS Arts and Science College, Coimbatore, Tamilnadu-641402 India; pbharathi36@gmail.com, kjagadeesh@yahoo.com

\begin{abstract}
We present a Modified Artificial Fish Swarm Algorithm (MFSA) which has many benefits that includes higher convergence rate, flexibility, fault tolerance and high accuracy. General behaviors systems of standard AFSA are: Prey, Follow, and Swarm. From the experimental results, we can say that our proposed system such as the optimized by Modified AFSA (MFSA) is better than that of PSO algorithm. Obviously, the feasibility of MAFSA based optimization method and the better global search capability of the AFSA have been proved.
\end{abstract}

Keywords: Data Mining, Particle Swarm Optimization, Modified Artificial Fish Swarm Algorithm, Minimal Support and Confidence, Global Search

\section{Introduction}

Data mining is an emerging technique to address the problem of reconstructing data into useful knowledge information from the user who can mine the results which they want. These rules are generated according to the knowledge by data mining algorithms in which one of most problematic steps in an association rule is discovery process knowledge validation. To solve this problem, association rules have been widely used in many application domains for finding patterns in data and to generate the association rules. The pattern discloses combinations of events that occur at the same time based on the interesting associations and/or correlation relationships among a large set of data items. The attributes value conditions will be shown by association rule that take place frequently together in a given dataset. Excluding the antecedent (the "if" part) and the consequent (the "then" part), an association rule has two important parameters which is used to gives the degree of uncertainty about the rule.

(i) Support: It is defined as the number of transactions that include all items in the antecedent and consequent parts of the rule. Finding frequent item sets with their supports above the minimal support threshold is called minimal support.

$\operatorname{Support}(\mathrm{X} \rightarrow \mathrm{Y})=\frac{\text { \# of transactions which contain } \mathrm{X} \& \mathrm{Y}}{\text { \# of transactions in the database }}$

(ii) Confidence: It is defined as the ratio of the number of transactions that contain all items in the consequent as well as the antecedent to the number of transactions that include all items in the antecedent. Using frequent item sets (found in support equation (1)) to generate association rules that have confidence levels above the minimal confidence threshold.

Confidence $(X \rightarrow Y)=\frac{\# \text { of transactions which contain } X \& Y}{\# \text { of transactions which contain } X}$

The objective of data mining is to find out significant associations along with items such that the occurrence of various items in a transaction will entail the occurrence of some other items. To accomplish this principle, suggested quite a lot of mining algorithms depends on the perception of large item sets to discover association rules in the

*Author for correspondence 
transaction data mining process into two stages. In the first stage, candidate item sets were produced and calculated through scanning the transaction data. The value is called as minimum support if the amount of an item set to emerge in the transactions be larger than a pre-defined threshold value then the item set is considered as a large item set. At the second stage the association rule, rules are generated from the first stage's result of large item sets. For each large item set all feasible association permutations were formed and the value is called as minimum confidence the output will be as association rules, when individuals calculated with confidence values larger than a predefined threshold.

The most representative association rule algorithm is the Apriori algorithm which is repeatedly generates candidate item sets and uses minimal support and minimal confidence to filter these candidate item sets to find highfrequency item sets ${ }^{1}$. Since the processing of the Apriori algorithm requires plenty of time, very important issue is a computational efficiency. To overcome the problem in Apriori, many researchers have proposed modified association rule-related algorithms. Savasere et al. introduced a partition algorithm for mining association rules which logically divides the database into a number of non overlapping partitions and it is demonstrated in ${ }^{2}$ by Krishna kumar. Toivonen introduced the sampling algorithm in $1996^{3}$. This algorithm is involved in finding association rules to reduce database activity. The technique generates accurate association rules, however in some cases some missing association rules might exist i.e., it does not generate all the association rules. The DIC algorithm was developed by Brin et al. ${ }^{4}$, DIC divides a database into various blocks marked by start points and frequently scans the database. A genetic algorithm is also having benefits for association rule Mining ${ }^{5}$. Genetic algorithms can create appropriate threshold values for association rule mining. In another study, an ant colony system was also employed to data mining under multi-dimensional constraints ${ }^{17}$ which is integrated with the clustering method to provide more precise rules ${ }^{18}$. Finally particle swarm optimization is proposed in R.J. Kuo ${ }^{7}$. In addition, binary particle swarm optimization is proposed in Sarath et $\mathrm{al}^{22}$ and PSO application for association rule mining is investigated in ${ }^{23}$, but in PSO algorithm feasible problem is occurred.

AFSA is one of the approaches stimulated both from the nature and swarm intelligence methods. AFSA was proposed by Li Xiao Lei in $2002^{8}$. This algorithm is an approach based on swarm behaviors that was inspired from social behaviors of fish swarm in the nature. This advance is significantly diverse from other methods which had only joined data mining and FSO together. AFSA is used in optimization applications e.g. PID controller parameters setting ${ }^{9}$ multi-objective optimization ${ }^{10}$, global optimization ${ }^{11}$, neural network learning ${ }^{12}$, data clustering $^{13}$, color quantization ${ }^{14}$ and etc. Most of their attempts had utilized to compact with the development of FSO as an optimization technique to resolve the data mining problems, for instance classification and clustering. To obtain good performance of the proposed algorithm planned to combine the local search method to perform globally best solution attained in each generation.

Because the processing of the Apriori algorithm needs abundance of time, its computational overhead is a very important drawback. With the intention of enhance the efficiency of Apriori; several researchers have proposed modified association rule-related algorithms. Park et al. proposed the DHP algorithm which can be derived from Apriori by introducing additional control. DHP makes use of an additional hash table that aims at limiting the generation of candidates as much as possible. DHP includes two major features, the efficient generation of large itemsets and the effective reduction of transaction database sizes. The Pincer-Search algorithm was proposed by Lin et al. and it can efficiently discover the maximum frequent set. This novel algorithm is analysed for Mining Multilevel Association Rule in ${ }^{19}$ by Usha Rani et al. The Pincer-Search algorithm integrates both the bottom-up and top-down directions. Very significant typical of the algorithm is that it is not necessary to clearly observe every frequent item set. Consequently, it performs well even when some maximal frequent item sets are long. The Pincer-Search algorithm can diminish both the number of times the database is read and the number of candidates deemed.

A single minimal support is insufficient for association rule mining since it cannot reflect the nature and frequency differences of the items in the database. Therefore, the Multiple Minimal Support algorithm ${ }^{20}$ was proposed by Liu et al. which is a more flexible and powerful model, allowing the user to specify multiple minimal item supports. This model enables us to find rare item rules with frequent items. Yang et al. proposed the improved hashbased scheme ${ }^{21}$, HMFS, for determining maximal frequent itemsets. The HMFS approach integrates the significance of both the DHP and the Pincer-Search algorithm. A genetic algorithm is also having benefits for association rule Mining ${ }^{5}$. Genetic algorithms can create appropriate threshold values for association rule mining. 


\section{Particle Swarm Optimization to Association Rule Mining}

In this work $^{7}$, Particle swarm optimization was proposed for association rule mining. The PSO algorithm has become an evolutionary computation approach and a significant heuristic method in recent years. Initialize PSO with a set of random particles (solutions) after that searches for optima is performed by updating generations. Each particle is updated by subsequent the two "best" values during all iterations. The first one is the best solution (fitness value) it has attained to this point and it is stored. This value is called "pbest". The other "best" value that is determined by PSO is the best value acquired as far as this by any particle. This best value is a global best and also it is referred as "gbest". Each particle updates its corresponding velocity and position after obtain these two best values.

The existing system includes two parts, preprocessing and mining. The overall process of this system is shown in Figure 1. The first part offers procedures which are used

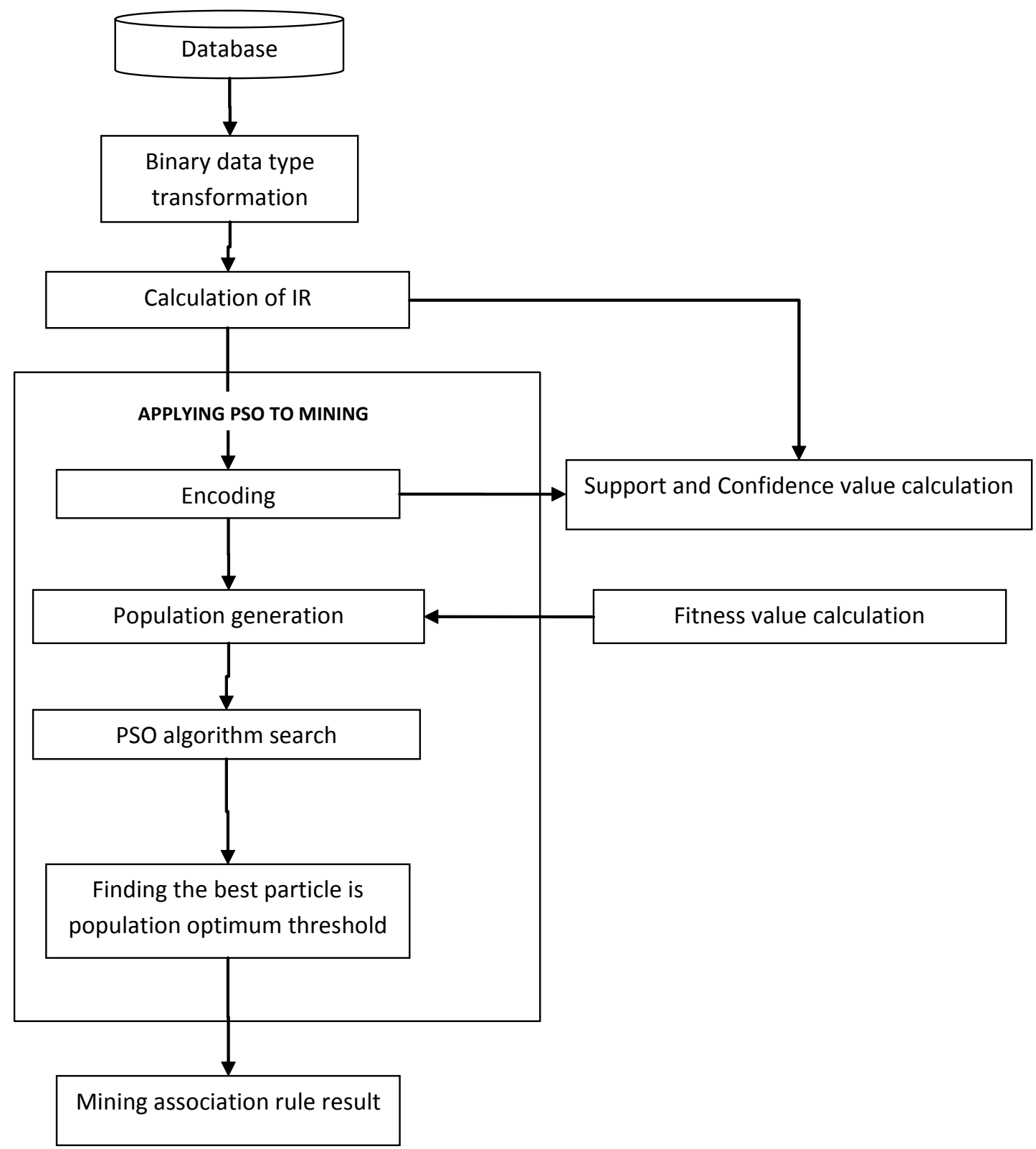

Figure 1. PSO based association rule mining. 
for estimating the fitness values of the PSO. In this part, the data are transformed and stored in a binary format. After that search range of the particle swarm is set with the help of the IR (item set range) value. The important goal of the system is the PSO algorithm is applied to mine the association rules are in the second part of the algorithm. This approach is used to transform transaction data into binary type data such as data is stored as either 0 or 1 . This scheme can speed up the database scanning operation, and it estimates support and confidence without difficulty and also faster.

When IR analysis is used to make a decision the rule length produced by chromosomes in particle swarm progression, search efficiency is improved. IR analysis is used to minimize the meaningless item sets in the process of particle swarm evolution. This approach addresses the front and back partition points of each chromosome, and the range decided by these two points is referred as IR. Thereafter, the IR value calculated is employed to choose the front and back partition points of the chromosomes. In the chromosome coding, the item set before the front partition point is called "item set X," while that between the front partition and back partition points is called "item set Y". The fitness value is used to estimate the significance of each particle. The fitness value of each particle calculated from the fitness function as shown in equation (3).

$$
\begin{aligned}
\text { Fitness }(\mathrm{k})= & \text { confidence }(\mathrm{k}) \times \log (\operatorname{support}(\mathrm{k}) \\
& \times \text { length }(\mathrm{k})+1)
\end{aligned}
$$

Where, Fitness $(\mathrm{k})$ is the fitness value of association rule type $\mathrm{k}$. Confidence $(\mathrm{k})$ is the confidence of association rule type k. Support (k) is the actual support of association rule type $\mathrm{k}$. Length $(\mathrm{k})$ is the length of association rule type $\mathrm{k}$.

The next step is population generation. To apply the evolution operation of the PSO algorithm, it is need to produce the initial population. We choose particles which have larger fitness values and that particles in this population are known as initial particles. First, the particle with the highest fitness value in the population is choosen as the "gbest." The initial velocity of the particle is set to be 0 , while the initial position is group of particles. The particle's initial "pbest" is its initial position and it is updated. Here designed a constrained technique which is used to estimate the distance between the particle's new position and all the possible particles. For complete particle evolution, the design of a termination condition is necessary. The evolution ends when the positions of all particles are unchanged. Another termination constraint occurs after 100 iterations and the evolution process of the particle swarm is concluded. As a final point, after the best particle is found, its support and confidence are suggested as the value of minimal support and minimal confidence.

\section{Modified Artificial Fish Swarm Algorithm (MAFSA)}

In the preliminary work $^{7}$, there is a problem the optimization. They are global optimization \& single optimization problem and feasibility problem in multidimensional function. To overcome these difficulties we propose a modified artificial fish swarm algorithm (MAFSA). AFSA is one of the swarm intelligence approaches which operate according to the population and stochastic search contributed to address optimization troubles. This algorithm has been used in various applications. AFs search the problem space by basic behaviors. AFSA objective function is defined as a food consistence degree in water area. At last, AFs reach to a point which its food consistence degree is highest (global optimum). In this algorithm basic behavior of AFSA is changed. The basic behaviors of AFSA are prey, follow, and swarm.

Overall architecture of our proposed system is shown in Figure 2. The proposed algorithm includes two parts, preprocessing and mining. The first part offers procedures which are used for estimating the fitness values of AFSA. In this part, the data are transformed and stored in a binary format. After that search range of the particle swarm is set with the help of the IR (item set range) value. The important goal of the system is the MAFSA algorithm is applied to mine the association rules are in the second part of the algorithm. Initially, we process with AFSA encoding, this step is related to chromosome encoding of genetic algorithms. Produce a population of AFSA based on the estimated fitness value is the next step. As a final point, the AFSA searching process takings until the condition is attained i.e., the best AF is found. The minimal support and minimal confidence are represented by the support and confidence of the best AF. Consequently, we can utilize this minimal support and minimal confidence for further association rule mining. 


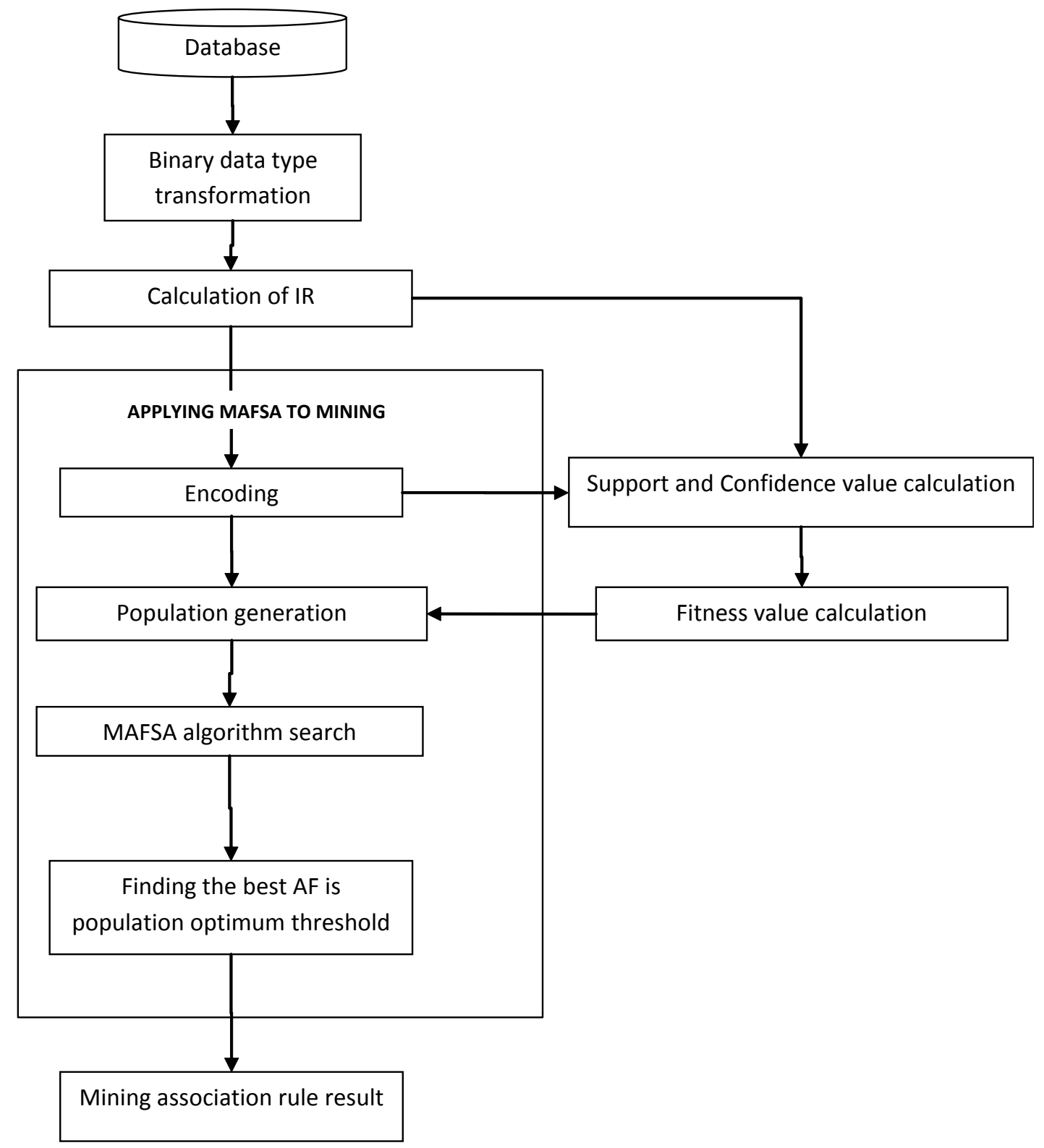

Figure 2. MAFSA based association rule mining.

\subsection{Binary Transformation}

Binary transformation ${ }^{6}$ is to converted transaction data into binary type data such as data is stored as either 0 or 1 . This scheme can speed up the database scanning operation, and it estimates support and confidence without difficulty and also faster. The transformation technique is discovered by five records such as $\mathrm{T} 1$ to $\mathrm{T} 5$, in the original data. Each data present in the records is transformed and stored as a binary type. For example, there are a total of only four various products in the database, thus four cells present in each transaction. Take B4 as an instance, this transaction only purchased products 2 and 3; as a result the values of cells 2 and 3 are both " $1 \mathrm{~s}$," in addition cells 1 and 4 are both " 0 s."

\subsection{Calculation of IR Value}

This study applies the AFSA in association rule mining, as well as in the calculation of IR value which is included in encoding process. The aim of such an insertion is to generate more significant association rules. Moreover, 
search efficiency is increased when IR analysis is utilized to decide the rule length generated by chromosomes in AFSA. IR analysis is used to minimize the meaningless item sets in the process of AF swarm evolution. This approach addresses the front and back partition points of each chromosome, and the range decided by these two points is referred as IR which is shown in equation (4),

$$
\begin{aligned}
\text { IR } & =[\log (\mathrm{m} \operatorname{TransNum}(\mathrm{m}))+\log (\mathrm{nTransNum}(\mathrm{n}))] \\
& =\frac{\operatorname{Trans}(\mathrm{m}, \mathrm{n})}{\operatorname{TotalTrans}}
\end{aligned}
$$

In the equation $4, \mathrm{~m} \neq \mathrm{n}$ and $\mathrm{m}<\mathrm{n}$. where " $\mathrm{m}$ " is defined as the length of the item set and $\operatorname{TransNum}(\mathrm{m})$ means the number of transaction records containing $\mathrm{m}$ products. " $n$ " is defined as the length of the itemset and TransNum(n) means the number of transaction records containing n products. Trans $(\mathrm{m}, \mathrm{n})$ is the number of transaction records purchasing $\mathrm{m}$ to $\mathrm{n}$ products. TotalTrans means the number of total transactions.

\subsection{Encoding}

Based on the general principle of association rule mining, the connection of the association rule of item set $\mathrm{X}$ to item set $\mathrm{Y}(\mathrm{X} \rightarrow \mathrm{Y})$ must be empty. Items which appear in the item set $\mathrm{X}$ do not appear on item set $\mathrm{Y}$, and vice versa. Thereafter, the IR value calculated is employed to choose the front and back partition points of the chromosomes. In the chromosome coding, the item set before the front partition point is called "item set X," while that between the front partition and back partition points is called "item set Y". In this work the chromosome encoding scheme is "string encoding". Each value represents a different item name, which means that item 1 is encoded as ' 1 ' and item 2 is encoded as ' 2 '. The representative value of each item is encoded into a string type chromosome by the consequent order.

\subsection{Fitness Value Calculation}

The fitness value is used to estimate the significance of each particle. The fitness value of each particle calculated from the fitness function as shown in equation (5),

$$
\begin{aligned}
\text { Fitness }(\mathrm{k})= & \text { confidence }(\mathrm{k}) \times \log (\operatorname{support}(\mathrm{k}) \\
& \times \operatorname{length}(\mathrm{k})+1)
\end{aligned}
$$

Fitness $(\mathrm{k})$ is the fitness value of association rule type $\mathrm{k}$. Confidence $(\mathrm{k})$ is the confidence of association rule type $\mathrm{k}$. Support $(\mathrm{k})$ is the actual support of association rule type $\mathrm{k}$. Length $(\mathrm{k})$ is the length of association rule type $\mathrm{k}$. The objective of this fitness function is maximization. The larger the AF support and confidence is the greater the strength of the association i.e., it is an important association rule. In the equation (5), parameters such as support, confidence and item set length must be estimated before determining the fitness value. This system uses the binary type data search approach.

\subsection{Population Generation}

In order to apply the evolution operation of the AFSA algorithm, it is need to produce the initial population. Here we choose AFs which have larger fitness values and that AFs in this population are known as initial AFs.

\subsection{Search the Best AF Process}

\subsubsection{Prey Behavior}

This behavior is an individual behavior. Besides processing this behavior, each AF does a local search around itself. By operating this behavior each AF efforts try number times to substitute to a new position with good fitness. Let assume $\mathrm{AF} i$ is in position $\mathrm{X}_{\mathrm{i}}$ and needs to carry out prey behavior. Subsequent steps are carried out in prey behavior:

- AF i considers a target position in visual by equation (6), then estimates its fitness value. $d$ is dimension number and Rand generates a random number with uniform distribution in $[-1,1]$ :

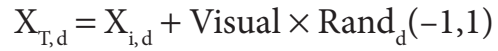

- If fitness value of position $\mathrm{X}_{\mathrm{T}}$ is better than current position of $\mathrm{AF} \mathrm{i}$, position will be updated by equation (7):

$$
\vec{X}_{\mathrm{i}}=\vec{X}_{\mathrm{T}}
$$

Steps $\mathrm{a}$ and $\mathrm{b}$ are performed try number times. By implementing above steps, in the best case, an AF can update its position at most try number times and move in the direction of improved positions. In the most terrible case, none of AF's efforts to determine better position will be achieved. After that in this condition after executing prey behavior, there will be no alternate at all.

\subsubsection{Follow Behavior}

This function is a group behavior which means interactions between swarm members are done globally. AFs 
in Standard AFSA in case of not determining improved positions in carry out standard prey behavior move one step indiscriminately ${ }^{15},{ }^{16}$ and drop their previous position. Although, in MAFSA, in prey behavior if an AF is not able to move to improved positions, it won't move at all and will stay its previous position. This reasons that the best $\mathrm{AF}$ (based on the fitness value) of swarm locates in the best found position by swarm member as far as this. The cause is that in prey behavior in MAFSA, an AF relocate if only moves to an improved position. In follow behavior, each of AFs moves one step toward the best AF of swarm by using equation (8):

$\vec{X}_{\mathrm{i}}(\mathrm{t}+1)=\vec{X}_{\mathrm{i}}(\mathrm{t})+\frac{\vec{X}_{\text {Best }}-\vec{X}_{i}(t)}{\text { Disis }_{i, \text { Best }}} \times[\operatorname{Visual} \times \operatorname{Rand}(0,1)]$

$\mathrm{X}_{\mathrm{i}}$ is position vector of $\mathrm{AF} \mathrm{i}$ which performs follow behavior and $\mathrm{X}_{\text {best }}$ is position vector of the best $\mathrm{AF}$ in swarm. As a result, AF i move at most to Visual extent in each dimension on the way to the best AF of swarm. In reality, after determining more food by a fish, other swarm members follow after it to reach more foods. Following the best AF of swarm causes convergence rate enhance and used to maintain reliability of AFs in a swarm.

\subsubsection{Swarm Behavior}

This behavior is a group behavior. In other words, it is processed globally among members of swarm. In swarm behavior, central position of swarm is estimated in terms of average of all swarm members position in each dimension. Central position of swarm $\mathrm{X}_{\text {center }}$ is obtained by:

$$
\mathrm{X}_{\text {center }, \mathrm{d}}=\frac{1}{N} \sum_{i=1}^{N} X_{i, d}
$$

As it is observed, component $d$ of vector $\mathrm{X}_{\text {center }}(9)$ is the arithmetic mean of component $d$ of all AFs of swarm. For AF i, move condition in the direction of central position is verified, i.e. $f\left(X_{\text {center }}\right) \geq f\left(X_{i}\right)$ and if this condition is satisfied, next position of AF $\mathrm{i}$ is obtained by:

$\vec{X}_{\mathrm{i}}(\mathrm{t}+1)=\vec{X}_{\mathrm{i}}(\mathrm{t})+\frac{\vec{X}_{\text {center }}-\vec{X}_{i}(t)}{\text { Dis }_{i, \text { center }}} \times[$ Visual $\times \operatorname{Rand}(0,1)]$

Equation (10) is used for all AFs that have worse positions than central position so they move toward $\mathrm{X}_{\text {center }}$ But for the best AF locating in $\mathrm{X}_{\text {Best }}$, if fitness value of $\mathrm{X}_{\text {center }}$ is better than $\mathrm{X}_{\text {Best }}$, next position of the best $\mathrm{AF}$ is obtained from:

$$
\vec{X}_{\text {Best }}=\vec{X}_{\text {center }}
$$

The reason of using (11) equation for the best $\mathrm{AF}$ is that it may be located in worse position than its current position by moving toward $\mathrm{X}_{\text {center }}$ by using corresponding equation, because it is possible to have worse positions in the way ending to $\mathrm{X}_{\text {center }}$ from $\mathrm{X}_{\text {Best }}$. Consequently, it may reason to lose the best position found by all members of swarm to this point. This problem is removed by using of above equation for the best AF. The cause of not using above equation for all AFs is that altering position of swarm fishes to an alike position guides to tremendous decrease in diversity of swarm and significant decrease of convergence rate.

\section{Experimental Result and Discussion}

The experiment conducted in the two datasets namely FoodMart2000 and stock market. In the first experiment, every transaction record in the FoodMart2000 has 1-13 items. Several kinds of transaction databases in FoodMart2000, so we only select sales fact 1997 data table for evaluation. In observe to data selection, 6000 customers are indiscriminately chosen along with their consequent transaction data at various times. After arrangement, there are a total of 12,100 transaction records for these customers. After calculating the IR values, we find that $\operatorname{IR}(1 \rightarrow 6)=5.86822$ is the largest. Therefore, we can generate five different dimensions of encoding types for the AF swarm. They are two dimensions, $1 \rightarrow 2$, three dimensions, $1 \rightarrow 3$ and $2 \rightarrow 3$, four dimensions, $1 \rightarrow 4,2 \rightarrow 4$, and $3 \rightarrow 4$, and five dimensions, $1 \rightarrow 5,2 \rightarrow 5,3 \rightarrow 5$, and $4 \rightarrow 5$, and six dimensions, $1 \rightarrow 6,2 \rightarrow 6,3 \rightarrow 6,4 \rightarrow 6$, and $5 \rightarrow 6$. According to these five dimensions, we can implement the AFSA mining process.

The second experiment investigates the association rules of investors' purchasing behavior of stocks within different industrial groups. The data are transformed into binary form with 1330 trading records. After the calculation of IR values, we find that $\operatorname{IR}(1 \rightarrow 2)=5.23184$ is the largest. Given the particle population of 10, this study implements the AFSA mining process and the final results show seven association item sets. They are \{Steel and Iron $\rightarrow$ Electronics\}, \{Tourism $\rightarrow$ Electronics\}, \{Wholesale and 
Retail $\rightarrow$ Electronics\}, \{Finance $\rightarrow$ Electronics $\},\{$ Chemicals $\rightarrow$ Electronics\}, \{Elec. Appliance and Cable $\rightarrow$ Electronics\} and \{Others $\rightarrow$ Electronics\}. According to the results of the association itemsets, investors who purchase stocks from the industrial groups including Steel and Iron, Tourism, Wholesale and Retail, Finance, Chemicals, Elec. Appliance and Cable and Others tend to purchase stocks from the Electronics industry.

In this experimentation we measure the performance of the system in terms of the association rule mining accuracy, computation time and rule quality. These parameters are measured by using the following equations. Data will be divided into two phases: training phase data and testing phase data. Generate a model based on the given rules in the target trouble using training data and afterward the model will be used on the testing data to attain the validation accuracy. The accuracy rate in data mining process is generally defined as:

Accuracy rate $=\frac{\text { True positive }+ \text { True negative }}{\text { True positive }+ \text { True negative }+ \text { False positive }+ \text { False negative }}$

The quality of the resulting rule is evaluated according to the rule-evaluation function

$$
\begin{gathered}
\text { Quality }=\text { sensitivity } \times \text { specificity } \\
=\frac{\text { True positive }}{\text { True positive }+ \text { False negative }} \times \frac{\text { True negative }}{\text { True negative }+ \text { False positive }}
\end{gathered}
$$

\section{- TP (True positive)}

If the outcome from a prediction is $\mathrm{p}$ and the actual value is also $\mathrm{p}$, then it is called a true positive (TP);

\section{- TN (True negative)}

A true negative (TN) has occurred when both the prediction outcome and the actual value are $\mathrm{n}$ in the number of input data.

\section{- FP (False positive)}

If the outcome from a prediction is $\mathrm{p}$ and the actual value is $n$ then it is said to be a false positive (FP).

\section{- FN (False negative)}

False negative $(\mathrm{FN})$ is when the prediction outcome is $n$ while the actual value is $\mathrm{p}$.

Figure 3 shows the accuracy rate of existing and proposed system in accordance to two parameters such as accuracy rate and the number of datasets. From the graph we can see that, when the number of datasets is increased then the accuracy rate of the system is reduced consequently. Accuracy rate of the system is reduced in existing system compared with the proposed system. Based on the comparison results which are extracted from the experimentation show the proposed technique works better than the existing schemes.

Figure 4 shows the quality rate of existing and proposed system in accordance to two parameters such as quality rate and the number of datasets. From the graph we can see that, when the number of datasets is increased then the quality rate of the system is reduced consequently. Quality rate of the system is reduced in existing system compared with the proposed system. Based on the comparison results which are extracted from the

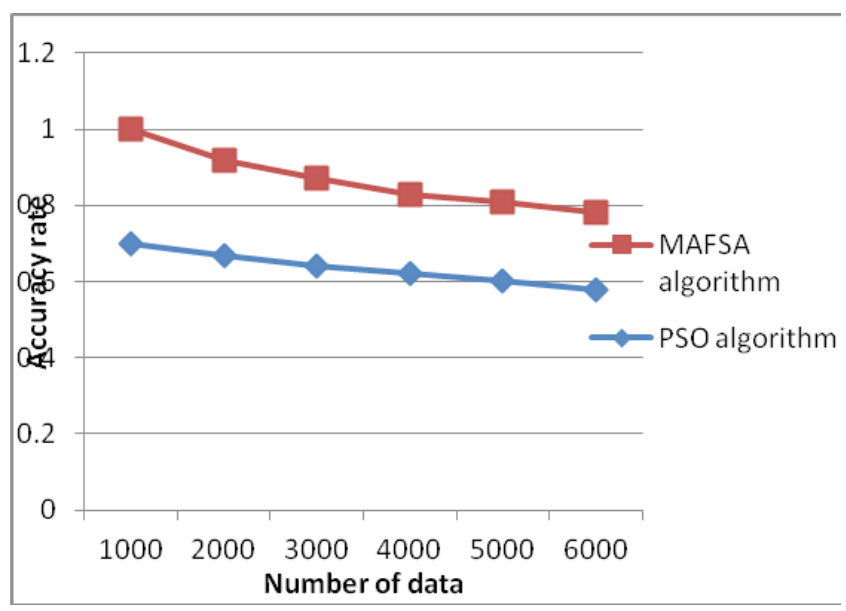

Figure 3. Accuracy comparison.

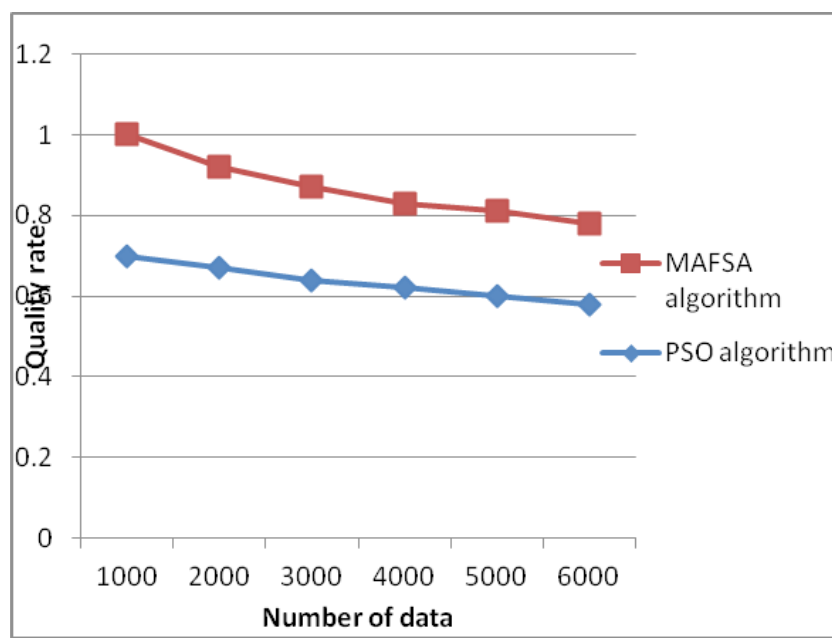

Figure 4. Quality comparison. 
experimentation show the proposed technique works better than the existing schemes.

Figure 5 shows relationship of the computation time rate between the existing and proposed system based on two parameters of population size and run time. Though the proposed MAFSA algorithm for association mining requires more computation time with increasing population size, the increase is not significant. From this graph we can say that, the computation time for the proposed algorithm less compared to the existing PSO algorithm. This means the proposed paper has more effective in computation time parameter.

\section{Conclusion}

Association rule mining is one of the most significant methods in the field of data mining. In our study, we are proposing the novel technique of Modified Artificial Fish Swarm Optimization Algorithm for the intension of enhance the association rule mining. This effective approach is utilized to overcome the problem on generating the association rules in the previous works. Here we have analysed the proposed technique through the comparison with the existing concept of association rule mining. Our proposed system offers a well-organized association rule mining application for the information searching from the outsized databases. Moreover, there are some related works to follow in the future. First, some work can be done to set a better initialization before the process of algorithm start. Second, adaptation of Visual with some learning methods will also enhance the effectiveness of the system so much.

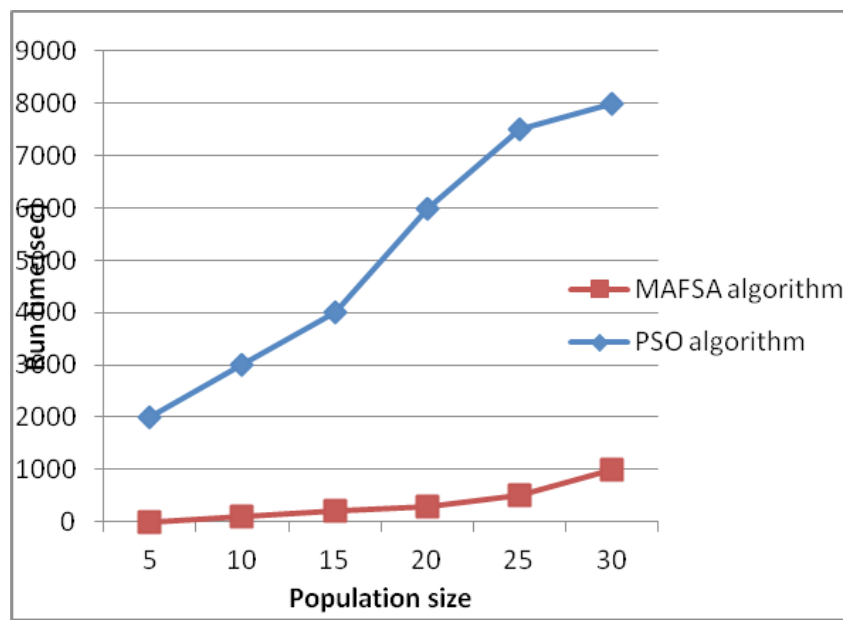

Figure 5. Computation time comparison.

\section{References}

1. Zaki M, Meira WJr. Data Mining and Analysis: Fundamental Concepts and Algorithms. Cambridge University Press; 2014 May. ISBN: 9780521766333.

2. Kumar AK, Amrita D, Priya NS. Mining Association Rules between Sets of Items in Large Databases. IJISME. 2013; 1(5):24-7. ISSN: 2319-6386

3. Toivonen H. Sampling large databases for association rules. Proceedings of the 22nd VLDB Conference; 1996; p. 134-45.

4. Birn S, Motwani R, Ullman JD, Tsur S. Dynamic itemset counting and implication rules for market basket data. Proceedings of the ACM SIGMOD. 1997; 255-64.

5. Jaiswal A, Dubey G. Identifying Best Association Rules and Their Optimization Using Genetic Algorithm. IJESE. 2013; 1(7):91-6. ISSN: 2319-6378.

6. Rao CS, Babu DR, Shankar RS, Kumar VP, Rajanikanth J, Sekhar CC. Mining Association Rules Based on Boolean Algorithm - a Study in Large Databases. International Journal of Machine Learning and Computing. 2013; 3(4):347-51.

7. Kuoa RJ, Chaob CM, Chiuc YT. Application of particle swarm optimization to association rule mining. Applied Soft Computing. 2011; 11:326-36.

8. Li LX, et al. An Optimizing Method based on Autonomous Animals: Fish Swarm Algorithm. Proc. of Systems Engineering Theory \& Practice. 2002.

9. Luo Y, et al. The Optimization of PID Controller Parameters Based on Artificial Fish Swarm Algorithm. 2007; 1058-62.

10. Jiang M, Zhu K. Multi objective optimization by Artificial Fish Swarm Algorithm. IEEE international conference on computer science and automation engineering; 2011.

11. Rocha AMAC, et al. An augmented Lagrangian fish swarm based method for global optimization. Journal of computational and applied mathematics. 2011; 235:4611-20.

12. Tsai HC, Lin YH. Modification of the fish swarm algorithm with particle swarm optimization formulation and communication behavior. Applied Soft Computing. Press, Corrected Proof, 2011.

13. He S, et al. Fuzzy Clustering with Improved Artificial Fish Swarm Algorithm. 2009; 317-21.

14. Yazdani D, et al. Color Quantization Using Modified Artificial Fish Swarm Algorithm. Australasian Conference on Artificial Intelligence; 2011; p. 382-91.

15. Branke J. The Moving Peaks Benchmark. Available at: http://people.aifb.kit.edu/jbr/MovPeaks/, 1999

16. Branke J. Evolutionary Optimization in Dynamic Environments. Kluwer Academic Publishers; 2001.

17. Kuo RJ, Shih CW. Association rule mining through the ant colony system for National Health Insurance Research Database in Taiwan. Computers and Mathematics with Applications. 2007; 54 (11-12):1303-18. 
18. Kuo RJ, Lin SY, Shih CW. Discovering association rules through ant colony system for medical database in Taiwan, to appear. International Journal of Expert Systems with Applications. 2007 Nov 3; 33.

19. Rani GU, Prakash RV, Govardhan A. Mining Multilevel Association Rule Using Pincer Search Algorithm. IJSR. 2013; 2(5):54-7. ISSN No: 2277-8179.

20. Liu B, Hsu W, Ma Y. Mining association rules with multiple minimal supports. Proceedings of the ACM SIGKDD International Conference on Knowledge Discovery \& Data Mining; 1999; San Diego, CA, USA. p. 337-41.

21. Yang DL, Pan CT, Chung YC. An efficient hash-based method for discovering the maximal frequent set.
Proceeding of the 25th Annual International Conference on Computer Software and Applications; 2001; p. 516-51.

22. Sarath KNVD, Ravi V. Association rule mining using binary particle swarm optimization. Engineering Applications of Artificial Intelligence. 2013 Sep; 26(8):1832-40.

23. Ankita S, Shikha A, Jitendra A, Sanjeev S. A Review on Application of Particle Swarm Optimization in Association Rule Mining. Proceedings of the International Conference on Frontiers of Intelligent Computing: Theory and Applications (FICTA), Advances in Intelligent Systems and Computing; 2013; 199:405-14. 